\title{
Aubele, Katharina: Vertriebene Frauen in der Bundesrepublik Deutschland. Engagement in Kirchen, Verbänden und Parteien 1945-1970 (Veröffentlichungen des Collegium Carolinum, Bd. 138), 484 S., Vandenhoeck \& Ruprecht, Göttingen 2018.
}

\section{Stephan Scholz}

Online publiziert: 7. Mai 2020

(C) Der/die Autor(en) 2020

„Ohne Frauen kann Integration nicht gelingen“, heißt es im aktuellen Bericht der Bundesbeauftragten für Migration, Flüchtlinge und Integration. Gezielt soll daher das weibliche „Empowerment“ gefördert werden. Mit einem ähnlichen, allerdings retrospektiv gewendeten Impetus möchte Katharina Aubele in ihrer 2015 an der Ludwig-Maximilians-Universität München als Dissertation angenommenen Arbeit die Frauen unter den deutschen Vertriebenen nach 1945 aus ihrem Erinnerungsstatus als lediglich passive Opfer lösen und stattdessen zeigen, „welche Leistungsfähigkeit““ (S. 1) sie als Akteurinnen in der Politik und Gesellschaft der Bundesrepublik entfalteten (die DDR bleibt ausgeblendet). Die Autorin verfolgt dabei einen frauengeschichtlichen Ansatz, der auf die „Sichtbarmachung von Frauen“ (S. 11) und die nachträgliche Würdigung ihrer Leistungen abzielt.

Konkret widmet sich Aubele in drei großen Teilen dem Engagement vertriebener Frauen im kirchlichen Bereich, in den Vertriebenenverbänden und in der Politik zwischen 1945 und 1970. In allen drei Bereichen geht es um die Handlungsmöglichkeiten der Akteurinnen, deren heimatliche Netzwerke durch Flucht und Vertreibung nach 1945 zunächst weggebrochen waren, um die Bedeutung ihres Vertriebenenhintergrundes für ihr Engagement und insbesondere um ihr Wirken für andere vertriebene Frauen. Der Grund für diese letzte Fokussierung bleibt unklar und vernachlässigt die Aktivität von Frauen in anderen Bezügen. Es wird nicht einsichtig, warum Politikerinnen, für die ,keine Aktivitäten in Bezug auf Vertriebene, Vertriebenenverbände oder Vertriebenenpolitik nachweisbar“ sind, nur „,der Vollständigkeit halber“ genannt werden, aber „keine Rolle für die Analyse“ spielen (S. 322) oder warum Frauen einer jüngeren Generation unberücksichtigt bleiben, die ,,ihrem Vertriebenenhintergrund keine große Relevanz beimaß und sich daher auch nicht für Vertriebene oder allgemein für die Angelegenheiten der Vertriebenen einsetzte“ (S. 393).

Diese perspektivische Verengung hat für den kirchlichen Bereich etwa zur Folge, dass Aubele nur Aktivitäten vertriebener Frauen für vertriebene Frauen untersucht.

S. Scholz (ه)

Carl von Ossietzky Universität Oldenburg, Oldenburg, Deutschland

E-Mail: stephan.scholz@uni-oldenburg.de 
In den Blick nimmt sie insbesondere Pfarrfrauen, Pfarrhelferinnen, Diakonissen und Fürsorgerinnen, die bereits vor 1945 als solche tätig waren und nachfolgend auf kirchliche Netzwerke aufbauen konnten, die meist schon vorher bestanden hatten und über den engeren Bereich ihrer Herkunftsregion hinausgingen. Der kirchliche Raum beider großen Konfessionen bot in den ersten Jahren nach Kriegsende vor allem in der sozialen Fürsorge Betätigungsmöglichkeiten.

Auch die Frauenarbeit in den Vertriebenenverbänden widmete sich in den ersten Jahren nach Auflösung des bis 1948 geltenden Koalitionsverbotes den sozialen Belangen von Frauen. Sie konzentrierte sich aber schon bald auf die kulturelle Brauchtumspflege und blieb, anders als die kirchliche Arbeit, einem ,,mehr oder weniger ausgeprägten Revanchismus verhaftet“ (S. 387 f.). Jenseits der Frauenarbeit, die von den männlichen Verbandsfunktionären vornehmlich rhetorisch anerkannt wurde, gelangten Frauen trotz wiederholter Versuche nicht in die Führungsebene der Vertriebenenverbände. Sie beteiligten sich allerdings immer wieder an den fortwährenden Konkurrenzkämpfen zwischen den verschiedenen Landsmannschaften und Landesverbänden. In der teilweise nur schwer nachvollziehbaren Rekonstruktion dieser Auseinandersetzungen und der ihnen zugrunde liegenden unübersichtlichen Verbandsstrukturen übernimmt Aubele gelegentlich zu stark die verbandliche Binnenperspektive. Immer wieder spricht sie von „den Vertriebenen“, wenn sie die Verbände meint, und übersieht dabei, dass diese nur ein bestimmtes Klientel unter den Vertriebenen vertraten.

Das zeigt sich nicht zuletzt im politischen Bereich, in dem ein nicht geringer Teil der Frauen mit Vertriebenenhintergrund Distanz zu den Vertriebenenverbänden wahrte. Insbesondere sozialdemokratische Politikerinnen sahen die personellen und konzeptionellen Kontinuitäten des (männlichen) Führungspersonals zur NSZeit kritisch. Aubele kann zeigen, dass für die Nähe zu den Verbänden weniger der Vertriebenenhintergrund entscheidend war als eine national-konservative politische Orientierung. Nicht nur in dieser Hinsicht bildeten die wenigen vertriebenen Frauen, die im Bundestag und in den Landtagen vertreten waren, eine höchst heterogene Gruppe, für die sich das Schicksal der Vertreibung als weniger gruppenbildend erwies als ihre jeweilige politische Ausrichtung.

Gerade im Teil zum politischen Engagement vertriebener Frauen zeigt sich der von Aubele favorisierte biografische Ansatz als nur bedingt ergiebig. Auf fast 50 Seiten werden Biogramme lediglich aneinandergereiht; die sich daran anschließende Typologisierung bleibt im Beschreibenden. Hier wie in der ganzen Arbeit macht sich eine zu starke Fixierung allein auf die Geschichte der Vertriebenen negativ bemerkbar. Eine Kontextualisierung in den größeren Zusammenhang geschlechtersensibler Politik- und Gesellschaftsgeschichte der Bundesrepublik erfolgt nicht. Die Berücksichtigung der entsprechenden Literatur zur allgemeinen Frauengeschichte hätte wichtige Vergleichsmöglichkeiten eröffnet, wenn es um die Bewertung der Handlungsmöglichkeiten von vertriebenen und nichtvertriebenen Frauen in Kirchen, Verbänden und Parteien geht.

Funding Open Access funding provided by Projekt DEAL.

Open Access Dieser Artikel wird unter der Creative Commons Namensnennung 4.0 International Lizenz veröffentlicht, welche die Nutzung, Vervielfältigung, Bearbeitung, Verbreitung und Wiedergabe in 
jeglichem Medium und Format erlaubt, sofern Sie den/die ursprünglichen Autor(en) und die Quelle ordnungsgemäß nennen, einen Link zur Creative Commons Lizenz beifügen und angeben, ob Änderungen vorgenommen wurden.

Die in diesem Artikel enthaltenen Bilder und sonstiges Drittmaterial unterliegen ebenfalls der genannten Creative Commons Lizenz, sofern sich aus der Abbildungslegende nichts anderes ergibt. Sofern das betreffende Material nicht unter der genannten Creative Commons Lizenz steht und die betreffende Handlung nicht nach gesetzlichen Vorschriften erlaubt ist, ist für die oben aufgeführten Weiterverwendungen des Materials die Einwilligung des jeweiligen Rechteinhabers einzuholen.

Weitere Details zur Lizenz entnehmen Sie bitte der Lizenzinformation auf http://creativecommons.org/ licenses/by/4.0/deed.de.

\section{Gassert, Philipp: Bewegte Gesellschaft. Deutsche Protestgeschichte seit 1945, 308 S., Kohlhammer, Stuttgart 2018.}

\section{Sven Reichardt}

Online publiziert: 25. März 2020

(c) Gesellschaft zur wissenschaftlichen Förderung politischer Literatur e.V. and the Author(s) 2020

Die äußerst lesenswerte Überblicksdarstellung des Mannheimer Zeithistorikers Philipp Gassert besticht durch ihre Thesenfreudigkeit. Neben zahlreichen Deutungsangeboten in neun chronologisch unterteilten Einzelkapiteln wartet diese Studie mit zwei übergreifenden Thesen auf. Erstens versteht Gassert Proteste als eine Form der politischen Kommunikation, die „weniger als Motor denn als Resonanzraum gesellschaftlichen Wandels“ (S. 273) zu begreifen ist. Zugleich verweist er darauf, dass dem Protest als gesellschaftlichem Kommunikationsmedium eine „Indikatorfunktion“ (S. 279) zukomme, welche die „Wahrnehmung gesellschaftlicher Defizite und Fehlentwicklungen “ (S. 279) besonders prägnant erfasse. Ob damit Proteste nun als Früh- oder doch eher als Spätwarnsysteme der Gesellschaft verstanden werden können, bleibt in dieser doppelten Perspektivierung offen. Weniger wichtig ist jedenfalls, so der Autor, die „Problemlösungskompetenz“ (S. 21) von Protesten im Vergleich zu der von ihnen ausgelösten gesellschaftlichen Resonanz. Ihre wichtigste

\footnotetext{
S. Reichardt $(\bowtie)$

Universität Konstanz, Konstanz, Deutschland

E-Mail: Sven.Reichardt@uni-konstanz.de
} 